\title{
A study of droplet evaporation
}

\author{
Koffi Sagna ${ }^{1}$, Amah D’Almeida ${ }^{1,2, *}$ \\ ${ }^{1}$ Laboratory of Solar Energy, Department of Physics, Faculty of Sciences, Université de Lomé, Lomé TOGO \\ ${ }^{2}$ Department of Mathematics, Faculty of Sciences, Université de Lomé, Lomé TOGO
}

\section{Email address:}

valmeida@univ-lome.tg (A. D’Almeida)

\section{To cite this article:}

Koffi Sagna, Amah D’Almeida. A Study of Droplet Evaporation. American Journal of Modern Physics. Vol. 2, No. 2, 2013, pp. 71-76. doi: 10.11648/j.ajmp.20130202.17

\begin{abstract}
We investigate the problem of the vaporization of a liquid droplet in a hotter environment of the same fluid. The Navier-Stokes equations are solved for a physical model which assumes spherical symmetry and laminar conditions in the quasi steady case. The study is mainly characterized by the fact that the equation of conservation of momentum is effectively taken into account and the velocity of the drop is not always uniform. Recession laws which are different from the classical $d^{2}$ law can be derived from the zeroth order approximation solution. Additional assumptions on the thermodynamical properties of the gas phase in subcritical conditions restore the classical law and permit the determination of an analytic expression for the vaporization ratio $\mathrm{K}$. The analysis of the evolution of the temperature, the density and the velocity in the droplet and in the gaseous phase reveals the existence of shock waves which develop from the center of the droplet towards its boundary and an unbalanced energetic layer attached to the interface when the velocity is not uniform in the drop.
\end{abstract}

Keywords: Drop Evaporation; $d^{2}$-Law; Quasi- Steady Recession; Mass Flow; Temperature

\section{Introduction}

The combustion and the evaporation of drops play a key role in the performance of internal combustion engines as gas turbines or rockets engines which conception is still based on empirical knowledge and a theory which does not enough take into account the complexity of the physical processes involved as well as the environment in the combustion chamber [1]. In these systems, the fuel is injected in the combustion chamber as a cloud of drops which vaporize and oxidize to release heat. The study of the evaporation of a drop isolated in an infinite environment, with diverse simplifying hypotheses, supplies important information for the understanding of these phenomena.

Following the pioneering works of Godsave [7] and Spalding [10], is developed a theoretical model which describes the process of evaporation of a drop in the subcritical condition. This model, which is termed "Quasi-Steady Model" and also called $d^{2}$-law, predicts that during the evaporation process, the droplet surface area, represented by the drop-squared diameter (the droplet being spherical), decreases linearly during its lifetime. Although this model is very successful in describing the vaporization process of fuel drop, the assumptions upon which the model has been developed are subjected to several experimental and nu- merical analyses [11], [12],[13]. Amongst the most controversial assumptions are those of constancy and uniformity of the macroscopic variables and the thermodynamic coefficients in the cold drop, the absence of convection and the steadiness of the gaseous phase which fits instantaneously the changes in the boundary conditions and the variation of the size of the drop. Concerning the first one of these assumptions, many numerical studies demonstrate the existence of an unsteady period of transition prior to the occurrence of the $d^{2}$-law. During this period, all the heat supplied to the drop is used to heat-up the liquid to its equilibrium temperature i.e. wet bulb temperature. Once this temperature is achieved, then all the heat transferred into the drop is used to gasify the liquid. Experimental and numerical results showed that the $d^{2}$ - law holds in the presence of convection but only once the drop reaches its thermal equilibrium. It must be recalled that the idea behind this assumption is to reduce numerical calculations and develop a rather simple analytical solution based on spherical symmetry. Regarding the third assumption, it was found that the $d^{2}$-law is no longer valid if the gaseous phase surrounding the droplet is unsteady [8],[11]. However its influence on this law is still not completely known. We determine in this work, by developing a formalism valid in subcritical conditions as well as in supercritical ones, recession laws of the radius of a drop of fluid suddenly introduced into a hotter environment of the 
same fluid and we compare them with the classic $d^{2}$-law in an attempt to clarify the questions raised in the previous paragraph. We then analyze the evolution of the macroscopic variables of the flow in the drop and in the gaseous phase.

In the section 2 we formulate the mathematical problem resulting from the modeling of the drop's vaporization phenomena by a spherically symmetrical drop evaporating in an infinite atmosphere initially in rest with uniform and constant pressure. After an asymptotic analysis we establish the quasi-steady equations. In the section 3 we solve analytically these equations and we establish recession laws, which in the general case differ from the classical $d^{2}$-law of the drop's radius. We deduce by making simplifying assumptions on the calorific capacity and the thermal conductivity of the gas, linear and nonlinear $d^{2}$-laws of time. Analytical solutions obtained in first approximation allow the study in the section 4 of the temperature, the density and the velocity in the drop and in the gaseous flow.

\section{Position of the Problem}

A drop of pure liquid of temperature uniformly equal to the temperature of saturated vapor at the subcritical pressure considered is abruptly introduced into a hotter infinite atmosphere initially in the rest. The radius, the density and the temperature of the drop at the initial moment are respectively $R^{\prime}(0)=R_{i n}^{\prime}, \rho^{\prime}(0)=\rho^{\prime}{ }_{\text {in }}$ and $T^{\prime}(O)=T^{\prime}{ }_{i n}$. The gaseous flow surrounding the drop is in a steady state characterized by a temperature $T_{\infty}^{\prime}$, a pressure $P_{\infty}^{\prime}$ and a density $\rho_{\infty}^{\prime}$. We suppose that there are neither external volume forces nor a forced convection, the initial spherical symmetry of the drop is thus preserved in the time.

We denote by $r^{\prime}$ the distance from the center of the drop and by $R^{\prime}$ the radius of the drop. A law of state (which will never be specified in the study because of the uniformity of the pressure) gives the pressure $P^{\prime}$ in the flow as function of the density $\rho^{\prime}$ and the temperature $T^{\prime} ; v^{\prime}$ is the velocity of the flow. The fluid is supposed perfect and we obtain the following system of conservation equations for the problem:

$$
\left\{\begin{array}{l}
\frac{\partial \rho^{\prime}}{\partial t^{\prime}}+\frac{1}{r^{\prime 2}} \frac{\partial\left(r^{\prime 2} \rho^{\prime} v^{\prime}\right)}{\partial r^{\prime}}=0 \\
\rho^{\prime}\left(\frac{\partial v^{\prime}}{\partial t^{\prime}}+v^{\prime} \frac{\partial v^{\prime}}{\partial r^{\prime}}\right)=0 \\
\frac{\partial T^{\prime}}{\partial t^{\prime}}+v^{\prime} \frac{\partial T^{\prime}}{\partial r^{\prime}}=\frac{1}{\rho^{\prime} C_{p}^{\prime} r^{\prime 2}} \frac{\partial}{\partial r^{\prime}}\left(r^{\prime 2} \lambda^{\prime} \frac{\partial T^{\prime}}{\partial r^{\prime}}\right)
\end{array}\right.
$$

We rewrite these equations in a reference moving with the boundary of the drop and we introduce the variable $x^{\prime}=$ $r^{\prime}-R^{\prime}$. We retain two characteristic times in the description of the phenomenon: the characteristic time of thermal diffusion $t^{\prime}{ }_{\text {dif }}$ and the drop's lifetime $t_{\text {res }}^{\prime}$. To take into account the gradient of density in the environment, we use two characteristic densities: $\rho_{l}^{\prime}$ in the drop and $\rho_{\infty}^{\prime}$ in the surrounding gas. We introduce the nondimensional num-

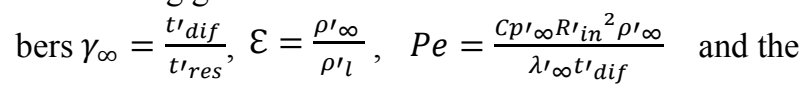

following nondimensional variables:

$$
\begin{aligned}
& t=\frac{t^{\prime}}{t_{\text {res }}^{\prime}}, r_{0}=\frac{R^{\prime}}{R_{\text {in }}^{\prime}}, x=\frac{x^{\prime}}{R_{\text {in }}^{\prime}}, v=\frac{v^{\prime} t_{\text {dif }}^{\prime}}{R_{\text {in }}^{\prime}}, \dot{r_{0}}=\frac{\dot{R}^{\prime} t_{\text {res }}^{\prime}}{R_{\text {in }}^{\prime}}, \\
& u=v-\gamma_{\infty} \dot{r}_{0}, T=\frac{T^{\prime}}{T_{\infty}^{\prime}}, \tilde{\rho}=\frac{\rho^{\prime}}{\rho_{\infty}^{\prime}}, \bar{\rho}=\frac{\rho^{\prime}}{\rho_{l}^{\prime}}
\end{aligned}
$$

Then the nondimensionl equations of the problem are given by:

$$
\left\{\begin{array}{l}
\frac{\gamma_{\infty}}{\epsilon}\left(\frac{\partial \bar{\rho}}{\partial t}+\frac{2 \bar{\rho} \dot{r}_{0}}{x+r_{0}}+\dot{r_{0}} \frac{\partial \bar{\rho}}{\partial x}\right)+\frac{1}{\left(x+r_{0}\right)^{2}} \frac{\partial\left[\left(x+r_{0}\right)^{2} \tilde{\rho} u\right]}{\partial x}=0, \\
\frac{\gamma_{\infty}}{\epsilon} \bar{\rho}\left(\frac{\partial u}{\partial x} \dot{r_{0}}+\gamma_{\infty} \ddot{r_{0}}\right)+\tilde{\rho}\left(\gamma_{\infty} \frac{\partial u}{\partial t}+u \frac{\partial u}{\partial x}\right)=0, \\
\frac{\gamma_{\infty}}{\epsilon}\left(\frac{\partial T}{\partial t}+\dot{r_{0}} \frac{\partial T}{\partial x}\right)+u \frac{\partial T}{\partial x}=\frac{1}{P e \tilde{\rho} C_{p}\left(x+r_{0}\right)^{2}} \frac{\partial}{\partial x}\left[\left(x+r_{0}\right)^{2} \lambda \frac{\partial T}{\partial x}\right]
\end{array}\right.
$$

with the initial conditions :

$$
\begin{gathered}
T(0, x)= \begin{cases}T_{\text {in }}(x), & x \leq 0 \\
T_{\infty}(x), & x>0\end{cases} \\
\rho(0, x)= \begin{cases}\rho_{\text {in }}(x), & x \leq 0 \\
\rho_{\infty}(x), & x>0\end{cases} \\
u(0, x)= \begin{cases}u_{\text {in }}(x), & x \leq 0 \\
u_{\infty}(x), & x>0,\end{cases}
\end{gathered}
$$

the boundary conditions :

$$
\begin{gathered}
T(t, x)=T_{l}(t, x), x \leq 0 \\
\lim _{x \rightarrow+\infty} T(t, x)=T_{\infty}(t) \\
\rho(t, x)=\rho_{l}(t, x), x \leq 0 \\
\lim _{x \rightarrow \infty} \rho(t, x)=\rho_{\infty}(t) \\
u(t, x)=u_{l}(t, x), x \leq 0 \\
\lim _{x \rightarrow+\infty} u(t, x)=u_{\infty}(t)
\end{gathered}
$$

and the jump relations :

$$
\begin{aligned}
& \rho_{l} u_{l}=\rho_{g} u_{g} \\
& \lambda_{l}\left(\frac{\partial T}{\partial x}\right)_{l}+\operatorname{Pe}_{\rho l} u_{l} \mathcal{L}_{0}=\lambda_{g}\left(\frac{\partial T}{\partial x}\right)_{g}, \mathcal{L}_{0}=\frac{h_{l}^{\prime}-h_{g}^{\prime}}{C p_{\infty}^{\prime} T_{\infty}^{\prime}}
\end{aligned}
$$

$h^{\prime}$ is the total enthalpy corresponding to the relative motion.

The use of two characteristic times and two characteristic densities in the dimensional analysis yields the two nondimensional numbers $\gamma_{\infty}$ and $\varepsilon$ and the thermal Peclet number $P e$ based on the characteristic quantities of the gas phase. The asymptotic studies conducted on this topic[2], [4], [9], generally use either $\gamma_{\infty}$ or $\varepsilon$ as small parameter. The form (3) of the nondimensional equations of conservation of the mass and the conservation of energy shows that unsteady phenomena are governed by the ratio $\tau=\frac{\gamma_{\infty}}{\varepsilon}$. In the equation of conservation of the momentum, it controls the terms related to the variation of the radius of the drop. It will be the small parameter of the asymptotic expansions. We notice that when one of the nondimensional numbers $\gamma_{\infty}$ or $\varepsilon$ is equal to one, we have the usual asymptotic expansions.

The effects due to the thermal expansion of the fluid and 
to the Stefan flow propagate in the gas with a characteristic speed very small relative to the speed of sound in the gas. So we can assert, for times that are very long compared to the characteristic acoustic time, that the pressure is uniform in the gaseous phase. We shall assume in the sequel that the pressure is uniform in all the flow. In first approximation, when this last assumption is taking into account, we obtain the system of the quasi- steady equations of the model:

$$
\left\{\begin{array}{l}
\frac{1}{\left(x+r_{0}\right)^{2}} \frac{\partial\left[\left(x+r_{0}\right)^{2} \rho_{0} u_{0}\right]}{\partial x}=0 \\
\rho_{0}\left(\gamma_{\infty} \frac{\partial u_{0}}{\partial t}+u_{0} \frac{\partial u_{0}}{\partial x}\right)=0 \\
u_{0} \frac{\partial T_{0}}{\partial x}=\frac{1}{P e \rho_{0} C p_{0}\left(x+r_{0}\right)^{2}} \frac{\partial}{\partial x}\left[\left(x+r_{0}\right)^{2} \lambda_{0} \frac{\partial T_{0}}{\partial x}\right] \\
\rho_{0}=\tilde{\rho}_{0}
\end{array}\right.
$$

In contrast with the classical formalism [2] the equation of conservation of the momentum is taken into account.

\section{The Drop Radius Recession Laws}

We integrate the first and the third equations of the system (11) and we find:

$$
\begin{aligned}
& \left(x+r_{0}\right)^{2} \rho_{0} u_{0}=f(t) \\
& \lambda_{0}(t, x) \frac{\partial T_{0}}{\partial x}(t, x)=\frac{k(t)}{\left.\left(x+r_{0}\right)^{2}\right)} \exp \left[\operatorname{Pe} \int_{0}^{x} \frac{C p_{0} \rho_{0} u_{0}}{\lambda_{0}}(t, a) d a\right]
\end{aligned}
$$

The gas is at rest, we thus have $u_{g}=-\gamma_{\infty} \dot{r_{0}}$. The jump relations (10) can then be written:

$$
\begin{aligned}
& \rho_{0 g} u_{0 g}=-\tau \dot{r}_{0} \\
& \lambda_{0 g}\left(\frac{\partial T_{0}}{\partial x}\right)_{g}=P e \rho_{0 g} u_{0 g} \mathcal{L}_{0}
\end{aligned}
$$

The relations (12) written on the boundary of the drop by taking into account the relation (13) give:

$$
f(t)=-\tau r_{0}^{2} \dot{r_{0}}=\frac{k(t)}{P e \mathcal{L}_{0}}
$$

The relation (14) gives the differential equation which describes the drop's radius evolution versus the time. It is however necessary to determine completely the function of time $k$ which depends upon the temperature to know the exact dependence law of the drop's radius of the time. We discuss the case where the heat capacity and the thermal conductivity are uniform and the case where they depend on time and on $x$ through the temperature.

\subsection{Uniform Heat Capacity and Thermal Conductivity : the $d^{2}$ Law}

We assume now that the quantities $\lambda_{0}$ and $C p_{0}$ do not depend explicitly on the space variable $x$. Then the integration of the second equation (12) gives:

$$
T_{0}(t, x)=\frac{k(t)}{P e C p_{0}(t) f(t)} \exp \left\{\frac{P e C p_{0}(t) f(t) x}{\lambda_{0}(t) r_{0}\left(x+r_{0}\right)}\right\}+\Theta(t)
$$

Using the boundary conditions we have:

$$
\begin{aligned}
& k(t)=\frac{P e C p_{0} f\left(T_{0}^{0}-T_{0}^{\infty}\right)}{1-\exp \left(\frac{P_{e} C p_{0} f}{\lambda_{0} r_{0}}\right)}, \Theta(t)=\frac{T_{0}^{\infty}-T_{0}^{0} \exp \left(\frac{P_{e} C p_{0} f}{\lambda_{0} r_{0}}\right)}{1-\exp \left(\frac{P_{e} C p_{0} f}{\lambda_{0} r_{0}}\right)} \\
& T_{0}^{0}(t)=T_{0}(t, 0), T_{0}^{\infty}(t)=\lim _{x \rightarrow+\infty} T_{0}(t, x)
\end{aligned}
$$

We substitute the expressions (16) of $k(t)$ in the relation (14) and obtain after computations the relation:

$$
r_{0}^{2}-1=-\frac{2}{\tau P_{e}} \int_{0}^{t} \frac{\lambda_{0}}{C p_{0}} \ln \left[1+\frac{C p_{0}\left(T_{0}^{\infty}-T_{0}^{0}\right)}{\mathcal{L}_{0}}(a)\right] d a
$$

which is a $\mathrm{d}^{2}$-law except the fact that the function of the second member is not a linear function of the time. When it is assumed that $L_{0}, \lambda_{0}$ and $C p_{0}$ are constant we obtain the classical $d^{2}$-law:

$$
r_{0}^{2}=1-K t, K=\frac{2 \epsilon \lambda_{0}}{P_{e} C p_{0} \gamma_{\infty}} \ln \left[1+\frac{C p_{0}\left(1-T_{0}^{0}\right)}{\mathcal{L}_{0}}\right]
$$

We can notice that the evaporation rate $K$ has an explicit analytical expression which is similar to the one found in [6], [7].

\subsection{Heat Capacity and Thermal Conductivity Depending on the Temperature}

When the thermal conductivity and the heat capacity depend on $t$ and $x$ through the temperature, the third equation (11) is expanded in the form:

$$
\frac{\partial^{2} T}{\partial x^{2}}+a \frac{\partial T}{\partial x}+b\left(\frac{\partial T}{\partial x}\right)^{2}=0, a=\frac{2}{x+r_{0}}-\frac{P_{e} C_{0} f(t)}{\lambda_{0}\left(x+r_{0}\right)^{2}}, b=\frac{1}{\lambda_{0}} \frac{\partial \lambda_{0}}{\partial T}(19)
$$

We infer from this:

$$
\frac{\partial T}{\partial x}=\frac{\exp \left[-\int_{0}^{x} a(t, v) d v\right]}{k(t)+J(t, x)}, J(t, x)=\int_{0}^{x} b(t, w) \exp \left[-\int_{0}^{w} a(t, v) d v\right] d w
$$

which gives by using the jump relations of the energy conservation equation:

$$
k(t) \dot{r}_{0}=-\frac{\lambda_{0}^{0}}{\tau \mathcal{L}_{0}}, \lambda_{0}^{0}(t)=\lim _{x \rightarrow 0^{+}} \lambda_{0}
$$

The expression of the function $k$ must therefore be determined to establish the recession law of the radius of the drop. By integrating the equation (20) we get the following relation:

$$
\frac{k(t)}{\lambda_{0}^{0}}=\frac{J(t, x)}{\lambda_{0}(T)-\lambda_{0}^{0}}
$$

Hence by making $x$ tend towards $+\infty$ we get:

$$
k(t)=\frac{\lambda_{0}^{0} J(t)}{\lambda_{0}^{\infty}-\lambda_{0}^{0}}, \lambda_{0}^{\infty}(t)=\lim _{x \rightarrow+\infty} \lambda_{0}[T(t, x)], J(t)=\lim _{x \rightarrow+\infty} J(t, x)
$$

It is difficult to obtain a completely analytical expression of the function $k$ which obviously depends on $r_{0}$ in the general case because of the form of $J(t)$. In the particular case where it assumed that $b$ and $\frac{C p_{0}}{\lambda_{0}}$ do not depend 
explicitly on $x$, we obtain:

$$
J(t)=\frac{r_{0}^{2}}{\alpha \lambda_{0}^{\infty}}\left(\frac{\partial \lambda_{0}}{\partial T}\right)_{\infty}\left[\exp \left(\frac{\alpha}{r_{0}}\right)-1\right], \alpha=\frac{P_{e} f(t) C_{0}^{\infty}}{\lambda_{0}^{\infty}},\left(\frac{\partial \lambda_{0}}{\partial T}\right)_{\infty}=\lim _{x \rightarrow+\infty} \frac{\partial \lambda_{0}}{\partial T}
$$

When we go back to dimensional variables, we have $\frac{\alpha}{r_{0}}=\frac{u_{0} r_{0}^{\prime}}{k^{\prime}}, k^{\prime}$ being the thermal diffusivity. In subcritical conditions $k^{\prime}$ is finite whereas $u_{0}^{\prime}$ and $r_{0}^{\prime}$ are very small; $\frac{\alpha}{r_{0}}$ is thus small and one can make the approximation $\exp \left(\frac{\alpha}{r_{0}}\right)-1 \cong \frac{\alpha}{r_{0}}$.

Substituting in (23) we have:

$$
k(t)=\frac{r_{0} \lambda_{0}^{0}}{\lambda_{0}^{\infty}\left(\lambda_{0}^{\infty}-\lambda_{0}^{0}\right)}\left(\frac{\partial \lambda_{0}}{\partial T}\right)_{\infty}
$$

Hence comparing with (21) we get the $d^{2}$-law

$$
r_{0}^{2}=1-\frac{2 \epsilon}{P e \gamma_{\infty}} \int_{0}^{t} \frac{\lambda_{0}^{\infty}\left(\lambda_{0}^{\infty}-\lambda_{0}^{0}\right)}{\mathcal{L}_{0}\left(\frac{\partial \lambda_{0}}{\partial T}\right)_{\infty}} d s
$$

We can notice that the function of time is always inversely proportional to the ratio $\frac{\gamma_{\infty}}{\varepsilon}$ and that the integrand depends only on the values of the thermodynamic coefficients at the interface and in the gaseous phase. If the thermodynamic coefficients are constant in the gas we have the classical $\mathrm{d}^{2-}$ law.

\section{Study of the Macroscopic Variables of the Flow}

The fact that the pressure is uniform allows to decouple the system of the conservation equations and to treat separately the system formed by the conservation equation of the mass and the conservation equation of the momentum and the conservation equation of the energy.

The equations (12) and (13) allow putting the system of the conservation equations of the mass and the momentum into the following form:

$$
\begin{aligned}
& \left(x+r_{0}\right)^{2} \rho_{0} u_{0}=f(t) \\
& \gamma_{\infty} \frac{\partial u_{0}}{\partial t}+u_{0} \frac{\partial u_{0}}{\partial x}=0
\end{aligned}
$$

The form of the second equation (27) suggests the research of the solution for the velocity in this form $u_{0}(t, x)=\frac{h(x)}{g(t)}$. We infer from this:

$$
\frac{d h}{d x}=c_{1}, \frac{d g}{d t}=\frac{c_{1}}{\gamma_{\infty}}
$$

The solution is thus singular in $\gamma_{\infty}$. We find for non ro $\gamma_{\infty}$ :

$$
u_{0}(t, x)=\frac{\gamma_{\infty}\left(c_{1} x+c_{2}\right)}{c_{1} t+\gamma_{\infty}}
$$

We infer from this, by using the mass quasi-steady equation, the expression of the density which is:

$$
\rho_{0}(t, x)=-\frac{h(t)\left(c_{1} t+\gamma_{\infty}\right)}{\left[x+r_{0}(t)\right]^{2}\left(c_{1} x+c_{2}\right) \epsilon}
$$

The solution (30) takes into account the variation of the relative velocity versus $x$ in the drop and in the gaseous phase. The boundary conditions on the interface are written for $e_{0}>0$ in the following form:

$$
\begin{aligned}
& \rho_{0}\left(t,-e_{0}\right)=\rho_{l}(t), u_{0}\left(t,-e_{0}\right)=u_{l}(t) \\
& \rho_{0}\left(t, e_{0}\right)=\rho_{g}(t), u_{0}\left(t, e_{0}\right)=u_{g}(t)
\end{aligned}
$$

They permit when $e_{0}$ tends towards zero, that is for $x=0$, to determine the function $h$, the velocity $u_{0 i}$ and the density $\rho_{0 i}$ at the interface so as to verify $\rho_{l} u_{l}=$ $\rho_{g} u_{g}=\rho_{0 i} u_{0 i}$. The equations (31) written at the initial instant $t=0$ allow to determine the constants $\mathrm{c} 1, \mathrm{c} 2$ and impose compatibility relations between the initial data on the interface. We so have, knowing that $r_{0}(0)=1$,

$$
\begin{aligned}
& h(t)=-\frac{\pi \dot{r}_{0}(t)\left[\left(r_{0}(t)-e_{0}\right)^{2}+\epsilon\left(r_{0}(t)+e_{0}\right)^{2}\right]}{2}, c_{1}=\frac{u_{g}(0)-u_{l}(0)}{2 e_{0}} \\
& c_{2}=\frac{u_{g}(0)+u_{l}(0)}{2}, \rho_{l}(0) u_{l}(0)=\rho_{g}(0) u_{g}(0) \frac{\left(1+e_{0}\right)^{2}}{\left(1-e_{0}\right)^{2}}
\end{aligned}
$$

The velocity is smooth in all the flow. But its profile varies in time. For the small values of time, it nullifies at the interface and changes sign up to the vanishing of the drop. Later it is constant in the flow (see Figure 1). The mass flow presents a shock which develops from the center of the drop towards its border. Its thickness and its lifetime depend on $\tau$ and the initial velocities of the drop and of the gas. An unbalanced energetic and mass layer remains attached to the border of the drop (see Figure 2, Figure 3).

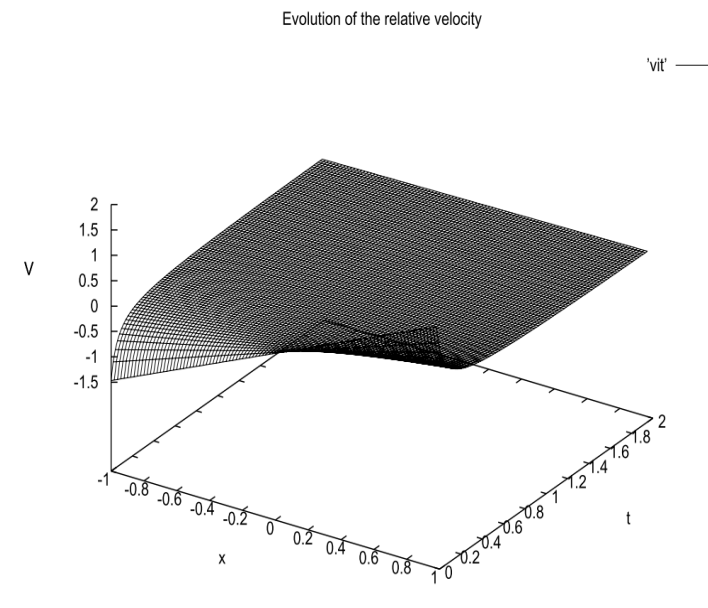

Figure 1. Evolution of the relative velocity.

When $\gamma_{\infty}$ is very small, the second equation (27) reduces to $\frac{\partial u_{0}}{\partial x}=0$. The relative velocity is thus independent of the distance from the interface when the lifetime of the drop is very great compared to the characteristic time of diffusion. The macroscopic variables are then given when the ther- 
modynamic coefficients are constant by:

$$
\begin{aligned}
& \rho_{0}(t, x)=\frac{-\tau r_{0}^{2} r_{0}}{\left(x+r_{0}\right)^{2} u_{0}} \\
& T_{0}(t, x)=\frac{k(t)}{P e C p_{0}(t) f(t)} \exp \left\{\frac{P e C p_{0}(t) f(t) x}{\lambda_{0}(t) r_{0}\left(x+r_{0}\right)}\right\}+\Theta(t) \\
& \Theta(t)=\frac{T_{0}^{\infty}-T_{0}^{0} \exp \left(\frac{P_{e} C p_{0} f}{\lambda_{0} r_{0}}\right)}{1-\exp \left(\frac{P_{e} C p_{0} f}{\lambda_{0} r_{0}}\right)} \\
& u_{0}(t)=\left\{\begin{array}{l}
u_{\text {in }}(t), x \leq 0 \\
u_{\infty}(t), x>0,
\end{array}\right.
\end{aligned}
$$

For this solution, the relative velocity in the drop and in the gas depends only on the time. But the temperature and the density depend on $t$ and on $x$. A shock wave whose characteristics depend on $\tau$ develops from the center of the drop towards its border. For small values of $\tau$ it is considerable only very close to the center of the drop and does not reach the border. For higher values of $\tau$ it can propagate in the entire drop and disappears in the neighborhood of the border. The mass flow is continuous on the border of the drop. Only the temperature presents an unbalanced boundary layer attached to the border.

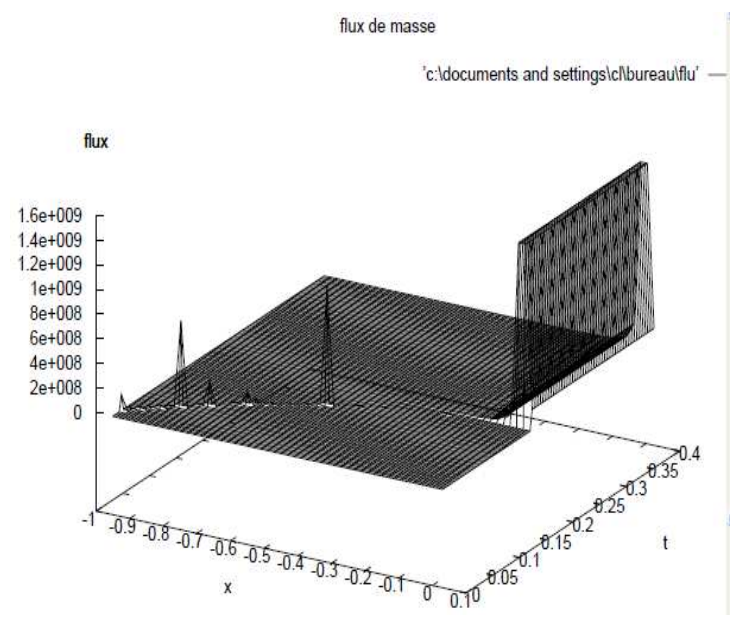

Figure 2. Evolution of the mass flow.

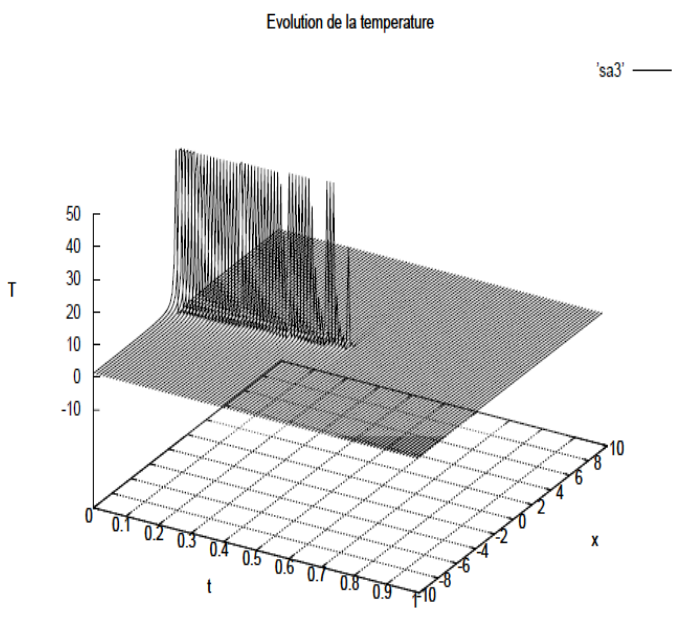

Figure 3. Evolution of the temperature.

\section{Conclusion}

We solved analytically the equations of Navier-Stokes for a subcritical drop in evaporation in quasi- steady conditions. The solution, obtained in first approximation yields recession laws that give the surface area or the squared-droplet diameter as a nonlinear function of time if the ratio $\frac{C_{p_{0}}}{\lambda_{0}}$ is independent of $x$ in the gaseous phase. We obtain the ${ }^{\lambda^{0}}$ classic $d^{2}$-law when the thermal conductivity and the heat capacity are constant in the gaseous phase. The functions of time established in the section 3 are the integrals of the functions $\lambda_{0}^{\infty}, C p_{0}^{\infty}$ which are the limits of $\lambda_{0}$ and $C p_{0}$ when $x$ tends towards $+\infty$ and $L_{0}$. When it is assumed that the flow of the gaseous phase is steady (and not quasi-steady) all these functions become constant and we have $d^{2}$-laws with linear functions of time.

Assuming that the $d^{2}$-law is verified, we determine the analytical expressions of the macroscopic variables of the flow and we analyze their evolution inside and outside the drop. We find that generally a shock wave develops from the center of the drop towards the border and that there is an energetic and mass unbalanced layer which remains attached to the border of the drop. The mass unbalanced layer does not exist when the relative velocity of the liquid phase is uniform. This is the case when the lifetime of the drop is very great compared to the characteristic time of diffusion in the gas.

\section{Acknowledgments}

This work began within the framework of a mission of research funded by the city of Paris. A. d'ALMEIDA thanks the city of Paris for its assistance.

\section{References}

[1] Harstad K., Bellan J., Isolated fluid oxygen drop behavior in fluid hydrogen at rocket chamber pressures,Int. J. Heat Mass Transfer,41, 3537-3550, 1998.

[2] Arias-Zugasti M., Garcia-Ybarra P., Castillo J. L., Unsteady Effects in Droplet Vaporisation Lifetimes at Subcritical and Supercritical Conditions, Combust. Sci. and Tech.,153,2000,179-191.

[3] Arias-Zugasti M., Garcia-Ybarra P., Castillo J. L., Droplet Vaporisation at critical Conditions :Long-time Convective-diffusive profiles along the critical isobar, Physical Review E,60, 3, 1999, 2930-2941.

[4] Préau S., Ouazzani J.,Zappoli B.,Prud'homme R., Supercritical density relaxation as a new approach of droplet vaporization, Phys. Fluids, 16 (11),2004,4075-4087.

[5] Prud'homme R., d'Almeida A., Sphères fluides avec injection, Colloque du GDR MFA, CD Rom CNES, 038490-64,2008.

[6] D'Almeida A., Prud'homme R., Evaporation de gouttes : lois de récession du rayon, $19^{\text {ème }}$ Congrès Français de Mécanique, Marseille, 24-28 août 2009. 
[7] Godsave G. A. E., Studies of the combustion of drops in a fuel spray. The burning of single drops of fuel, in Fourth Symposium on Combustion, The Combustion Institute (ed), $1953,818-830$.

[8] Waldman C., Theory of non-steady state droplet combustion, Proc. Combust. Inst., 15, 429, 1971.

[9] Sanchez-Tarifa C., Crespo A., Fraga E.,Theoretical model for the combustion of droplets in super-critical conditions and gas pockets, Astronautica Acta, 17,685-692, 1972.

[10] Spalding D. B.,Combustion of fuel particles, Fuel,30, 1951.

[11] Law C. K., Chung S. H., Srinivasan N.,Gas phase qua- si-steadyness and fuel vapor accumulation effects in droplet burning, Combust. Flame,38,173,1980.

[12] S. Saengkaew, T. Charinpanikul, C. Laurent, Y. Biscos, G. Lavergne, G. Gouesbet,and G. Grehan, "Processing of individual rainbow signals", Exp. Fluids, vol. 48,pp. 111-119, 2010 .

[13] S. Saengkaew, D. Bonin, P. Briard, and G. Gréhan, "Réfractométrie d'arc-en-ciel global à faisceau pulsé : Estimation des concentrations et des distancesinter-particulaires", in Congrès Francophone de Techniques Laser (CFTL2010), Vandoeuvre-lès-Nancy (France), 14-17 septembre 2010. 